\title{
Mice null for the deubiquitinase USP18 spontaneously develop leiomyosarcomas
}

Fadzai Chinyengetere ${ }^{1,6,7}$, David J. Sekula ${ }^{1,6,7}$, Yun Lu ${ }^{1,6,7}$, Andrew J. Giustini ${ }^{6,7,8}$, Aarti Sanglikar $^{5}$, Masanori Kawakami ${ }^{1,6,7}$, Tian Ma M, ${ }^{1,7}$, Sandra S. Burkett ${ }^{9}$, Burton L. Eisenberg ${ }^{4,5,6,7}$, Wendy A. Wells ${ }^{3,5,6,7}$, Paul J. Hoopes ${ }^{4,5,6,7,8}$, Elizabeth G. Demicco ${ }^{10}$, Alexander J Lazar ${ }^{11,12}$, Keila E. Torres ${ }^{12,13}$, Vincent Memoli ${ }^{3,5,6,7}$, Sarah J. Freemantle $e^{1,6,7}$ and Ethan Dmitrovsky ${ }^{1,2,5,6,7,14^{*}}$

\begin{abstract}
Background: USP18 (ubiquitin-specific protease 18) removes ubiquitin-like modifier interferon stimulated gene 15 (ISG15) from conjugated proteins. USP18 null mice in a FVB/N background develop tumors as early as 2 months of age. These tumors are leiomyosarcomas and thus represent a new murine model for this disease.

Methods: Heterozygous USP18 +/- FVB/N mice were bred to generate wild-type, heterozygous and homozygous cohorts. Tumors were characterized immunohistochemically and two cell lines were derived from independent tumors. Cell lines were karyotyped and their responses to restoration of USP18 activity assessed. Drug testing and tumorigenic assays were also performed. USP18 immunohistochemical staining in a large series of human leiomyosacomas was examined.

Results: USP18 -/- FVB/N mice spontaneously develop tumors predominantly on the back of the neck with most tumors evident between 6-12 months (80 \% penetrance). Immunohistochemical characterization of the tumors confirmed they were leiomyosarcomas, which originate from smooth muscle. Restoration of USP18 activity in sarcoma-derived cell lines did not reduce anchorage dependent or independent growth or xenograft tumor formation demonstrating that these cells no longer require USP18 suppression for tumorigenesis. Karyotyping revealed that both tumor-derived cell lines were aneuploid with extra copies of chromosomes 3 and 15. Chromosome 15 contains the Myc locus and MYC is also amplified in human leiomyosarcomas. MYC protein levels were elevated in both murine leiomyosarcoma cell lines. Stabilized P53 protein was detected in a subset of these murine tumors, another feature of human leiomyosarcomas. Immunohistochemical analyses of USP18 in human leiomyosarcomas revealed a range of staining intensities with the highest USP18 expression in normal vascular smooth muscle. USP18 tissue array analysis of primary leiomyosarcomas from 89 patients with a clinical database revealed cases with reduced USP18 levels had a significantly decreased time to metastasis $(P=0.0441)$.
\end{abstract}

Conclusions: USP18 null mice develop leiomyosarcoma recapitulating key features of clinical leiomyosarcomas and patients with reduced-USP18 tumor levels have an unfavorable outcome. USP18 null mice and the derived cell lines represent clinically-relevant models of leiomyosarcoma and can provide insights into both leiomyosarcoma biology and therapy.

Keywords: USP18, ISG15, Leiomyosarcoma, Murine cancer model

\footnotetext{
* Correspondence: ethan.dmitrovsky@dartmouth.edu

'Department of Pharmacology and Toxicology, Dartmouth, Hanover, NH,

USA

${ }^{2}$ Department of Medicine, Dartmouth, Hanover, NH, USA

Full list of author information is available at the end of the article
}

(c) 2015 Chinyengetere et al. Open Access This article is distributed under the terms of the Creative Commons Attribution 4.0 International License (http://creativecommons.org/licenses/by/4.0/), which permits unrestricted use, distribution, and reproduction in any medium, provided you give appropriate credit to the original author(s) and the source, provide a link to the Creative Commons license, and indicate if changes were made. The Creative Commons Public Domain Dedication waiver (http://creativecommons.org/publicdomain/zero/1.0/) applies to the data made available in this article, unless otherwise stated. 


\section{Background}

Soft tissue sarcomas are heterogeneous malignancies of mesenchymal origin. They arise from supporting tissues of the body including adipose, muscle and fibrous connective tissue [1]. Although relatively rare, with an international incidence ranging from 1.8 to 5 cases per 100,000 per year, approximately $50 \%$ of patients succumb to this malignancy [2-4]. Median survival for patients with advanced or metastatic sarcoma is only 12 months [5-7]. There are currently limited therapeutic options for those diagnosed with recurrent or advanced stage sarcoma. Surgery and radiation are the mainstays of treatment with doxorubicin being the most frequently used single chemotherapeutic agent, providing only $10-30 \%$ objective responses, but no increase in survival $[8,9]$. Hence, there is a need to identify new therapeutic approaches for these diverse tumors.

Leiomyosarcomas arise from smooth muscle and belong to the subgroup of sarcomas that have a complex, unbalanced karyotype, usually harboring non-specific genetic alterations and chromosomal instability $[10,11]$. Consistent with other sarcomas with complex karyotypes, leiomyosarcomas frequently deregulate P53 expression [12-14]. Effective targeted therapy for the complex karyotypeassociated sarcomas is likely hindered by their tumor heterogeneity. If there were a way to identify subsets of sarcomas with specific genetic alterations, this would improve the classification or treatment outcomes for these malignancies. For instance, polysomy of chromosome 8 in humans (syntenic to polysomy 15 in mice) is reported in complex sarcomas including leiomyosarcomas $[15,16]$. Notably, $M Y C$ is found on chromosome 8 and is a frequently overexpressed oncogene in human cancers. Indeed, MYC overexpression in a subset of leiomyosarcomas was associated with decreased metastasis-free survival [17]. There are few tractable mouse models that mimic the histopathology and molecular characteristics of human leiomyosarcomas. New mouse models could be instrumental in therapeutic development.

Ubiquitin-specific protease 18 (USP18) is a deubiquitinase for an interferon-regulated ubiquitin-like process, ISGylation [18]. This is the conjugation of interferon (IFN)-stimulated gene 15 (ISG15), a 15kDa ubiquitin-like moiety, to diverse target proteins $[19,20]$. USP18 knockout mice were initially generated on a C57Bl/6 and 129 mixed background [21]. These mice displayed neurological symptoms and hydrocephalus and did not survive beyond 5 months [21]. Subsequent generation of USP18 knockout mice on a pure $\mathrm{C} 57 \mathrm{Bl} / 6$ background found homozygous deletion of USP18 was embryonic lethal [22]. In contrast, this phenotype was not seen in the USP18 knockout FVB background mice. C57Bl/6-129-USP18 knockout mice were also hypersensitive to type I IFN as seen when they were treated with the IFN-inducer, poly-
IC; this was fatal for USP18 knockout, but not for the wild-type mice [23]. Recently, human ISG15 deficiency was found to cause a decrease in USP18 accumulation and this was hypothesized to cause the loss of negative feedback of type I interferon signaling in these patients leading to auto-inflammation [24].

USP18 overexpression is associated with augmented oncogene or growth factor receptor expression such as the epidermal growth factor receptor (EGFR) and tumorpromoting effects in acute promyelocytic leukemia, kidney and lung cancer [25-28]. Intriguingly, we report here the previously unrecognized development of spontaneous subcutaneous sarcomas, histopathologically diagnosed as leiomyosarcoma, in FVB-USP18 knockout mice. These murine sarcomas recapitulate critical characteristics of human leiomyosarcoma including aneuploidy, overexpression of MYC and deregulation of P53.

USP18 analysis of clinical leiomyosarcoma revealed abundant staining in normal smooth muscle cells, which was retained by some sarcomas, but lost in others. While loss of USP18 did not significantly impact overall survival or disease-free survival, it did significantly decrease the time to metastasis indicating a key role for USP18 levels in leiomyosarcoma clinical biology.

Cell lines were independently derived from sarcomas that arose in different mice. These sarcomas were histopathologically diagnosed as leiomyosarcomas. Cell lines were aneuploid and overexpressed MYC relative to mouse embryonic fibroblasts (MEFs). These lines formed rapidly growing subcutaneous sarcomas following transplantation into athymic and immunocompetent mice. These murine sarcoma cell lines, when coupled with the parental USP18 null mice, comprise tractable models to accelerate the discovery and development of new therapies for human leiomyosarcoma.

\section{Methods \\ Mice}

FVB-USP18 heterozygous mice were purchased from the Jackson Laboratory. These mice were bred to generate FVB-USP18 knockout mice. Generation of FVB-USP18 knockout mice is previously described [21, 29]. Genotyping for the knockout was done using polymerase chain reaction (PCR) assays according to a previously published protocol from the Jackson Laboratory (\#007225). Mice were bred in a non-pathogen environment, according to an Institutional Animal Care and Use Committee (IACUC)-approved protocol at Dartmouth. All mice experiments were done in accordance with this protocol. Animals are housed in cages with a 68 inch squared area with no more than 4 adult mice per cage. They live with a $12 \mathrm{~h}$ light/12 $\mathrm{h}$ dark light schedule at a humidity of $30-70 \%$ and a temperature of $72+1-2{ }^{0} \mathrm{~F}$. Mice receive ad libitum food (Teklad Irradiated Rodent Diet 2918) 
and water. Bedding is hardwood chip and at a depth of 0.25 inches.

Once tumors were detected in USP18 null mice, animals were closely monitored three times per week and weighed. Mice with greater than $10 \%$ body weight loss or with a tumor that was causing substantial clinical symptoms were euthanized using IACUC-approved procedures.

\section{Tumor tissues}

Acquisition of tissue specimens and clinical information and subsequent analyses were approved by the Institutional Review Board (IRB) of The University of Texas MD Anderson Cancer Center (UTMDACC) and Dartmouth Hitchcock Medical Canter (DHMC). Patients provided written informed consent.

\section{Generation of USP18 knockout cell lines and cell culture}

Two independent cell lines (designated KHC-1and KHC-2) were generated from subcutaneous tumors, using described methods [30]. Cells were cultured on tissue culture plates in RPMI-1640 media with $10 \%$ fetal bovine serum (FBS). These cells have been continuously cultured for up to 40 passages at $5 \% \mathrm{CO}_{2}$ and at $37^{\circ} \mathrm{C}$ in a humidified incubator. Cell karyotyping analysis confirmed these cells were of murine origin and immunohistochemical analysis established they retained expression of antigens consistent with the original tumors. SK-LMS-1 human leiomyosarcoma cells were purchased from ATCC where cell identity is confirmed using standard short tandem repeat (STR) analyses. SK-LMS-1 cells were cultured in Eagle's Minimum Essential medium (EMEM).

The siRNAs for knockdown experiments were from Ambion. MYC siRNA \#1 (\#s70224), MYC siRNA \#2 (\#s70226) and negative control (\#4390843) were individually transfected into cells using Lipofectamine 2000 (Invitrogen) according to the manufacturer's instructions and with triplicate replicate transfections. Protein was collected and analyzed by immunoblot assays to verify knockdown $24 \mathrm{~h}$ post-transfection. To quantify MYC protein knockdown, densitometry readings of immunoblots (from 3 independent transfections) were measured using NIH ImageJ analyses. Cell growth was assessed 72 $\mathrm{h}$ post-transfection using the CellTiter-Glo assay (Promega). Growth comparisons were normalized to cells transfected with control siRNA.

\section{Reconstitution of USP18 in sarcoma cell lines}

Lipofectamine 2000 (Invitrogen) was used to transfect the pRetroX-IRES-ZsGreen1-USP18 retroviral vector or an empty retrovirus (Clontech) into Plat-A retroviral packaging cells according to manufacturer's protocol (Cell Biolabs). Viral supernatants were collected after 48 h. KHC-1 and KHC-2 cells were transduced with the respective viral supernatants in regular culture media supplemented with polybrene $(4 \mathrm{~g} / \mathrm{mL})$ (Sigma). Green fluorescent protein expressing cells were sorted after 48 h using a FACStar Plus cytometer (Becton Dickinson).

\section{Drug treatments, proliferation and apoptosis assays}

KHC-1, KHC-2 and human leiomyosarcoma SK-LMS-1 cells were each treated with doxorubicin $(0.2 \mu \mathrm{M})$ or with interferon-beta (IFN $\beta$ ) (500 units/mL) (Sigma). Cell growth was assayed using a CellTiter-Glo Luminescent assay (Promega) in four independent, replicate experiments (each performed in at least triplicates). Unless stated otherwise, growth was measured after $72 \mathrm{~h}$. Apoptosis was analyzed using Annexin V:FITC and PI positivity in flow cytometry assays according to the manufacturer's instructions (AbD Serotec). All experiments were performed at least 3 times.

\section{Immunoblot analyses}

Cells were lysed in modified radioimmunoprecipation buffer (RIPA) [31] supplemented with protease and phosphatase inhibitors (Sigma). Proteins were resolved on SDSPAGE gels and transferred to nitrocellulose membranes (primary antibodies listed in Additional file 1: Table S1). Membranes were blocked in tris-buffered saline with $0.1 \%$ Tween 20 (TBS/Tween) plus $5 \%$ non-fat milk powder. Primary antibodies were diluted in this blocking buffer or in $5 \%$ bovine serum albumin (BSA) in TBS/Tween. Primary antibodies were detected with horseradish peroxidase (HRP) conjugated secondary antibodies (Santa Cruz and GE Healthcare) and visualized using Luminata Forte chemiluminescent HRP detection reagents (Millipore).

\section{Anchorage independent growth}

Cells were plated in 6 well plates $\left(10^{4}\right.$ cells/well $)$ in $0.5 \%$ agarose, as before [31]. The agar and cell suspensions were layered onto a $1 \%$ base agarose in RPMI-1640 media containing 10\% FBS. After formation of the top agar, $1 \mathrm{~mL}$ of RPMI-1640 medium containing 10\% FBS was overlaid before incubation at $37^{\circ} \mathrm{C}$ in a $5 \% \mathrm{CO}_{2}$ humidified incubator for 14 days. Colonies greater than five cells were counted using a microscope in four independent experiments.

\section{Orthotopic sarcoma model}

Cells for subcutaneous flank implantation into nude mice were resuspended in a 1:1 mixture of phosphate buffered saline (PBS) and reduced growth factor Matrigel (BD Biosciences). The cell number used for athymic nude mice (01B74/Athymic NCr-nu/nu, NCI) was $2.7 \times$ $10^{5}$ for the KHC-1 line, $1 \times 10^{5}$ for KHC-2 cells and for syngeneic NCr-FVB (NCI) mice this was $1 \times 10^{5} \mathrm{KHC}-1$ cells. Four 8 week old female mice were used per cell line for subcutaneous tumor growth analysis and were randomly assigned the different cell lines. Mice were weighed and tumor size was measured biweekly with calipers. The orthotopic mouse tumor experimental 
protocol was reviewed and approved by Dartmouth's Institutional Animal Care and Use Committee (IACUC). Mice were sacrificed using an IACUC-approved protocol when tumor diameter measured $15 \mathrm{~mm}$ or if mice became symptomatic and/or cachectic (loss of $10 \%$ or more of body weight). Tumor volume was calculated using the formula $0.5 \times$ length $\times(\text { width })^{2}$ where width is defined as the smallest diameter.

\section{Histopatology}

Routine hematoxylin and eosin ( $\mathrm{H} \& \mathrm{E})$ and immunostaining were performed as described in the Additional file 2 .

\section{Statistics}

Two-tailed $t$ tests were used for statistical analysis unless otherwise noted. Statistical significance was defined as ${ }^{*} P<0.05$ and ${ }^{* *} P<0.01$. For tissue microarray USP18 immunohistochemical scores were grouped based on comparison to normal muscle as $1+$ (low) or 2-3+ (retained). This scoring system is previously described [32]. For primary soft tissue tumors only, the method of Kaplan and Meier was used to assess outcomes in relation to USP18 expression. Univariable and multivariable Cox proportional hazards regression models were used to estimate associations between USP18 expression and overall survival (OS), disease-specific survival (DSS) and time to first metastasis, with alpha of 0.05 considered as significant.

\section{Results}

\section{FVB-Usp18 knockout mice develop leiomyosarcomas}

USP18 knockout mice on an FVB background were generated and their genotypes confirmed by PCR assays (Fig. 1a). About $80 \%$ of FVB-USP18 knockout mice developed clinically-detectable subcutaneous tumors in the trunk or neck regions (Fig. 1b and c). This was in contrast to wild-type and heterozygous USP18 mice where no such tumors were detected (Fig. 1c). In some USP18 knockout mice, dystrophic calcifications appeared with or without tumor development (Additional file 1: Figure S1). $H$ \& E staining of formalin-fixed sections of these murine tumors revealed anaplastic tumors with numerous mitotic bodies and a spindle-like morphology (Fig. 1d). Some of the tumors had giant cells (Fig. 1d). The dystrophic calcification could be a precursor lesion for these tumors. Calcification is often seen in leiomyomas, which are benign smooth muscle cell malignancies, but has also been seen in leiomyosarcomas [33].

The sarcomas occurred in USP18 null mice as early as 2 months of age (Table 1). Younger mice (less than 6 months old) tended to exhibit more dystrophic calcifications unlike the gross tumor development in mice older than 6 months (Additional file 1: Figure S1A). Most of the USP18 knockout mice exhibited palpable sarcomas at ages from 6 months to 12 months (Table 1 and Fig. 1b). While there were more male than female mice displayed in Table 1, this was not due to a sex-linked difference in sarcoma development. The percentage of mice that developed sarcomas was similar for males and females.

To further characterize these sarcomas, histopathological analyses were performed. These sarcomas were of mesenchymal origin because they were typically negative for the epithelial marker cytokeratin (Table 1). S100, a marker for melanomas and some neural-derived tumors was absent in every sarcoma except for one with rare nuclear staining. Of the mesenchymal-specific cell markers, all 13 of the tumors stained diffusely positive for smooth muscle actin. For desmin, 12 of 13 stained positively with 1 negative and 1 with rare staining. Calponin staining was the lowest of the three mesenchymal markers examined and it exhibited diffusely positive staining in 5 tumors and focal staining in another 5; 2 tumors stained negatively and another tumor was not evaluable (Table 1). All the tumors analyzed were classified as high-grade leiomyosarcomas and these sarcomas exhibited necrosis. A representative sarcoma and sarcoma derived cell line are shown in Fig. 1e and f. For the cell line shown in Fig. If and for some of the other sarcomas there was some heterogeneity of staining with calponin and desmin.

Mice lacking the tumor suppressor P53 spontaneously develop tumors, specifically lymphomas and sarcomas [34]. Studies of clinical leiomyosarcomas have shown frequent deregulation of P53 [12]. In this study, 5 of 13 sarcomas showed abundant staining for P53 indicating stabilization and deregulation of this protein. Figure 1 shows a tumor with abundant nuclear P53 and another that was negative for P53 staining (Fig. 1g).

\section{Restoration of USP18 expression in USP18 null leiomyosarcoma cell lines}

Leiomyosarcoma cell lines were derived from two different mice with sarcomas and were designated as KHC-1 and KHC-2, respectively (Fig. 2a). Loss of the USP18 gene in each cell line was confirmed by PCR assay genotyping. To determine if restoring USP18 activity reversed tumorigenicity in these cell lines, each was retrovirallytransduced with either human USP18 or an empty vector. USP18 functional reconstitution was demonstrated by decreased ISG15 conjugates in response to IFN $\beta$ treatment versus parental and empty vector control cells (Fig. 2a).

There was no statistically significant difference in monolayer or soft agar growth assays between KHC-1 and KHC-2 cells independently stably transfected with an empty vector or the USP18 expression vector (Fig. 2b and c). When these leiomyosarcoma cell lines were each 
transplanted into athymic mice $(n=4)$, palpable tumors arose within 2 to 3 days of subcutaneous implantation. Tumors grew at a similar rate and reached their predetermined study endpoint diameter of $15 \mathrm{~mm}$ within 10-20 days (data not shown). Engrafted tumors were harvested from athymic mice and USP18 expression in the USP18 stably transfected tumor-forming cells was detected (Additional file 1: Figure S1B). Tumor histology was similar to the sarcomas in USP18 null mice. There was no statistically significant difference observed in tumor growth between cells transduced with an empty vector versus those with restored USP18 activity (Fig. 2d).
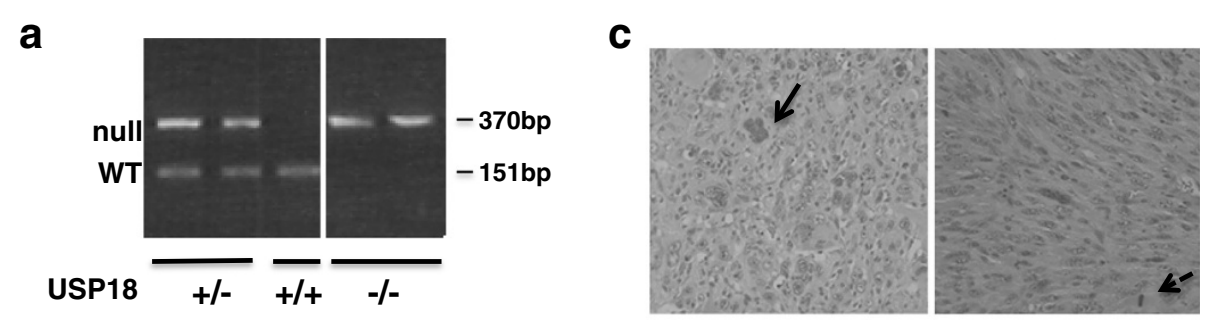

b

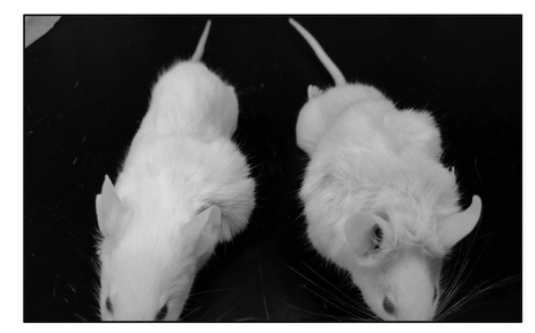

d
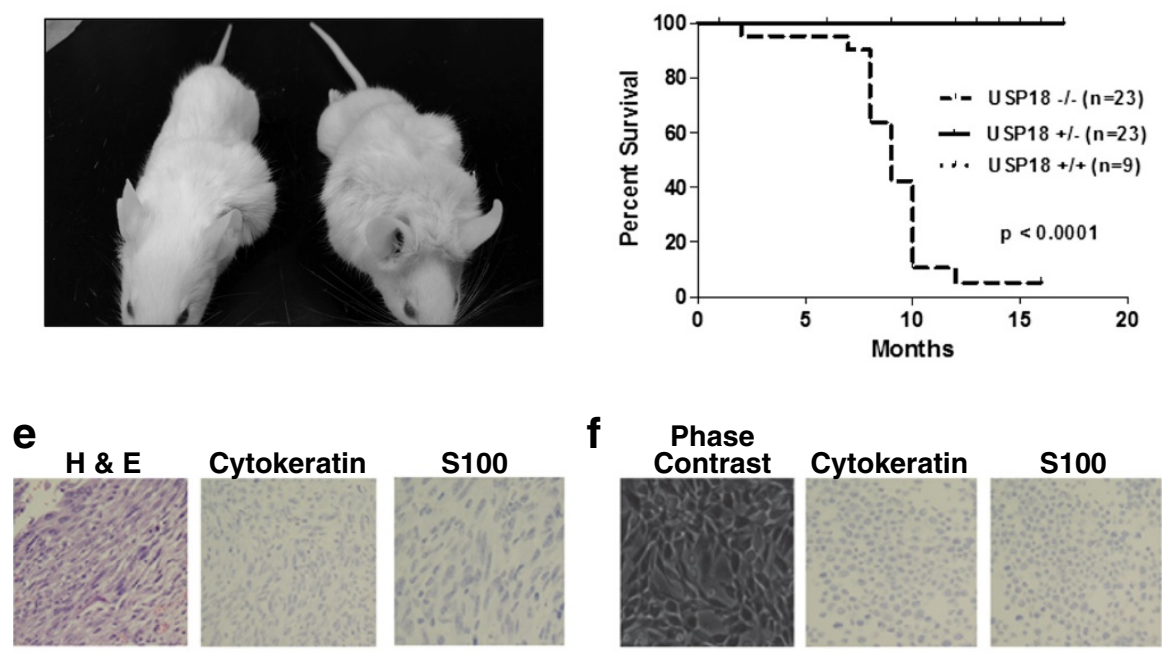

f
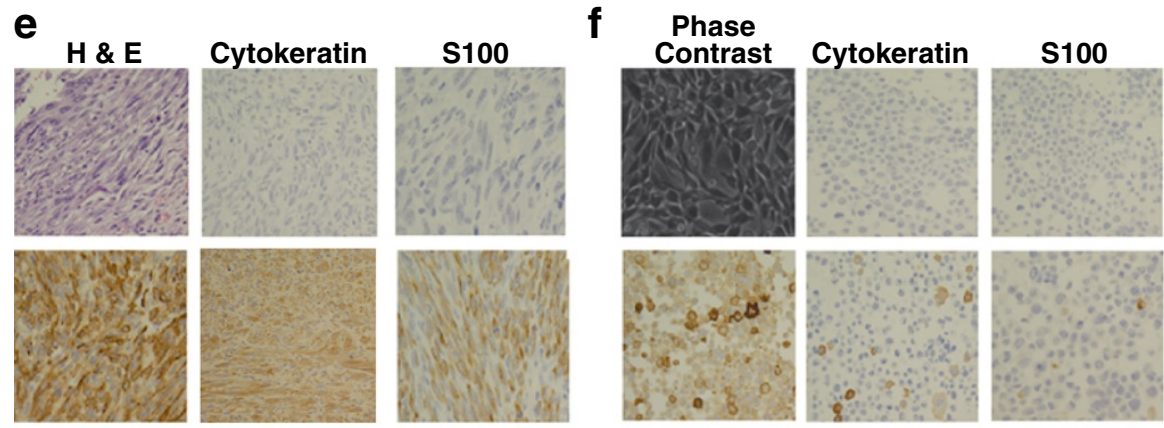

SMA

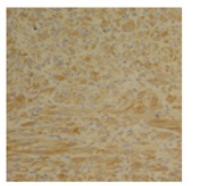

Calponin

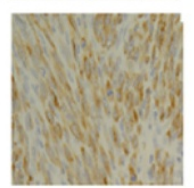

Desmin

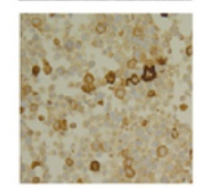

SMA

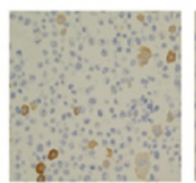

Calponin

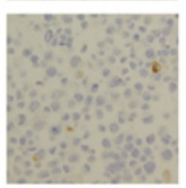

Desmin

g

P53

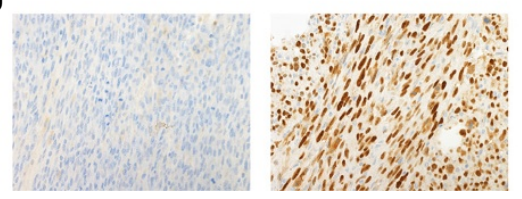

Fig. 1 FVB-USP18 knockout mice develop sarcomas. a: PCR genotyping assays of USP18-/-, USP18+/-, and USP18+/+ genomic DNA (null allele, $370 \mathrm{bp}$ and wild-type allele $151 \mathrm{bp}$ ). b: USP18 null mice with tumors. c: H \& E staining of representative sarcomas. The solid arrow indicates a multinucleate giant cell. The spindle cell characteristic of the sarcomas is depicted in the right panel and the hatched arrow indicates a mitotic cell. d: Kaplan-Meier curve is displayed depicting the times when sarcoma development necessitated euthanasia in USP18-/- mice versus USP18+/+ and USP18+/- mice, respectively. USP18 null mice significantly developed sarcomas $(P<0.0001)$ while no sarcomas were seen in wild-type or heterozygous mice. e: $\mathrm{H}$ \& E staining of a representative murine leiomyosarcoma in a USP18 -/- mouse is displayed along with the expression profiles for S100, cytokeratin (AE1/AE3), desmin, calponin and SMA (smooth muscle actin). f: A representative leiomyosarcoma cell line (KHC-2) was also examined for the same markers displayed in panel E. A phase-contrast image of this cell line is provided. $\mathbf{g}$ : The P53 immunostaining is of two USP18 -/- murine leiomyosarcomas, one positive for nuclear P53 and one negative 
This experiment was repeated in immunocompetent $\mathrm{FVB} / \mathrm{N}$ mice and there was no difference in tumor growth (or immune infiltrates) detected between cells transplanted with restored USP18 activity and controls $(n=4)$. Tumor onset and immune infiltrates were similar to that observed in athymic mice (data not shown). Hence, an intact immune system did not affect murine leiomyosarcomas growth. While loss of USP18 preceded sarcoma formation, these sarcoma cell lines did not depend on loss of USP18 expression for survival, growth or tumorigenicity.

\section{USP18 null leiomyosarcoma cell lines are aneuploid and overexpress MYC}

Since leiomyosarcomas can exhibit aneuploidy [10, 11], cytogenetic analyses were performed on KHC-1 and KHC-2 cell lines. Standard karyotyping by G-banding and spectral karyotyping (SKY) were used to characterize the cells and both were aneuploid (Fig. 3a and b). The KHC-1 cell line had trisomy 3 , tetrasomy 15 , deletions of chromosomes 3 and 4 and a derivative chromosome 9. KHC-2 cells showed trisomy 3,12 and 15, trisomies with derivatives on 8 and 16, and a translocation of chromosomes 6 and 11 (Fig. 3b). Chromosome 15 contains the loci for $M y c$ (orthologous to a chromosome 8 region in humans). Chromosome 15 is also the site of two proto-oncogenes of the Wnt family, Wnt1 and Wnt10b [35]. Aneuploidy is frequent in clinical leiomyosarcomas and the chromosomal sites shown here are homologous to those altered in human leiomyosarcomas $[15,16]$.
Given that both cell lines exhibited polysomy in chromosome 15, which carries the Myc locus (Fig. 3a and b), MYC protein levels were examined in $\mathrm{KHC}-1$ and $\mathrm{KHC}-2$ cells. Compared to wild-type mouse embryonic fibroblasts (MEFs) and NIH-3T3L1 cells, MYC protein levels were higher in the leiomyosarcoma cell lines (Fig. 3c). To determine the effect of MYC repression in these cell lines, two different MYC siRNAs were individually transfected into each cell line and growth effects were compared with controls after $72 \mathrm{~h}$. Knockdown of MYC protein in each cell line was confirmed (Fig. 3d) with MYC protein levels decreased to between 12-27 \% and $25-35 \%$ of controls in KHC-1 cells and $\mathrm{KHC}-2$ cells, respectively, as determined by densitometry from 3 independent experiments. Reduced MYC expression modestly, yet significantly inhibited growth of both cell lines (Fig. 3e and f).

\section{IFN $\beta$ and doxorubicin treatments of murine and human leiomyosarcoma cell lines}

USP18 knockout mice on a C57Bl/6 background were hypersensitive to type I IFN [23]. Given this, we sought to treat USP18 null sarcoma cell lines with IFN $\beta$ to determine if they exhibited type I IFN hypersensitivity. Growth inhibition occurred 24, 48 and $72 \mathrm{~h}$ after IFN $\beta$ treatment, with the most prominent inhibition at $72 \mathrm{~h}$ (Fig. 4a and b, left panels). Both of these leiomyosarcoma lines exhibited apoptosis after IFN $\beta$ treatments (Fig. $4 \mathrm{a}$ and $b$, right panels). Independent treatments of a human leiomyosarcoma cell line with identical IFN $\beta$ and

Table 1 Molecular pathology of the murine sarcomas

\begin{tabular}{|c|c|c|c|c|c|c|c|}
\hline Mouse \# (M/F) & Age (months) & $\mathrm{S} 100$ & Cytokeratin & SMA & Desmin & Calponin & $\mathrm{p} 53$ \\
\hline $1(\mathrm{M})$ & 9 & 0 & 0 & $3+$ & $2+$ & focal-1+ & $3+$ \\
\hline $2(M)$ & 10 & 0 & rare $2+$ & $3+$ & $2+$ & $3+$ & 0 \\
\hline $3(M)$ & 8 & 0 & rare $2+$ & $3+$ & $3+$ & 0 & $1+$ \\
\hline $4(M)$ & 10 & Rare nuclei & rare $1+$ & $3+$ & $2+$ & $2+$ & $1+$ \\
\hline $5(M)$ & 9 & 0 & 0 & $3+$ & 3 & focal-1+ & 0 \\
\hline $6(F)$ & 7 & 0 & 0 & $2+$ & 0 & $N A^{*}$ & 0 \\
\hline $7(F)$ & 9 & 0 & 0 & $3+$ & rare $2+$ & $3+$ & $3+$ \\
\hline $8(M)$ & 10 & 0 & 0 & $2+$ & $1+$ & focal-1+ & $3+$ \\
\hline $9(F)$ & 10 & 0 & 0 & $2+$ & $3+$ & 0 & 0 \\
\hline $10(M)$ & 9 & 0 & rare $1+$ & $2+$ & $3+$ & $2+$ & $2+$ \\
\hline $11(\mathrm{M})$ & 8 & 0 & 0 & $3+$ & $3+$ & $2+$ & $1+$ \\
\hline $12(M)$ & 2 & 0 & 0 & $1+$ & $1+$ & focal-2+ & 0 \\
\hline $13(\mathrm{M})$ & 9 & 0 & 0 & $2+$ & $2+$ & focal-1+ & $3+$ \\
\hline $\mathrm{KHC}-1$ & N/A & 0 & 0 & $3+$ & $2+$ & focal-2+ & $\times$ \\
\hline KHC-1 & $\mathrm{N} / \mathrm{A}$ & 0 & 0 & $3+$ & $2+$ & $2+$ & $\times$ \\
\hline
\end{tabular}

This Table provides scoring for the different marker antibodies used to diagnose the histopathology of these sarcomas. The markers include S100, cytokeratin (AE1/AE3), smooth muscle actin (SMA), desmin, calponin and p53. All staining was diffuse unless otherwise noted. N/A refers to not applicable. The abbreviations $M$ and $F$ refer to male and female, respectively

$N A^{*}$ is Not Evaluable and $x$ is Not Done 
doxorubicin concentrations conferred modest growth inhibition (Additional file 1: Figure.S3). Due to the slower growth characteristics of this cell line, it cannot be assumed that the USP18 null cells are hypersinsitive to IFN $\beta$. Treatment of the murine USP18 null cell lines with restored USP18 activity did not alter IFN $\beta$ response. This indicated that USP18 levels did not affect IFN $\beta$ sensitivity in these cells when grown in vitro (Additional file 1: Figure.S4).

Immunoblot analyses of the murine cell lines uncovered constitutive phosphorylation of STAT3, which is oncogenic in other systems [36]. With development of inhibitors of the upstream kinase Janus kinase 2 (JAK2), it is possible to target this pathway. $\mathrm{KHC} 1$ and $\mathrm{KHC} 2$ cells were each growth inhibited by the JAK2-STAT3 pathway inhibitor JSI-124 (Additional file 1: Figure. S2) at doses that inhibited JAK2-STAT3 signal transduction (Additional file 1: Figure. S2C). These sarcoma cell lines were also sensitive to clinically achievable doxorubicin dosages that caused decreased proliferation and increased apoptosis in these cells (Fig. 4c and d). Thus, these new murine leiomyosarcoma cell lines established antineoplastic activity of known and new agents for treatment of leiomyosarcomas.

\section{USP18 expression in human leiomyosarcomas}

To establish the translational research relevance of these pre-clinical findings, USP18 immunohistochemical studies were performed in 21 different human leiomyosarcomas

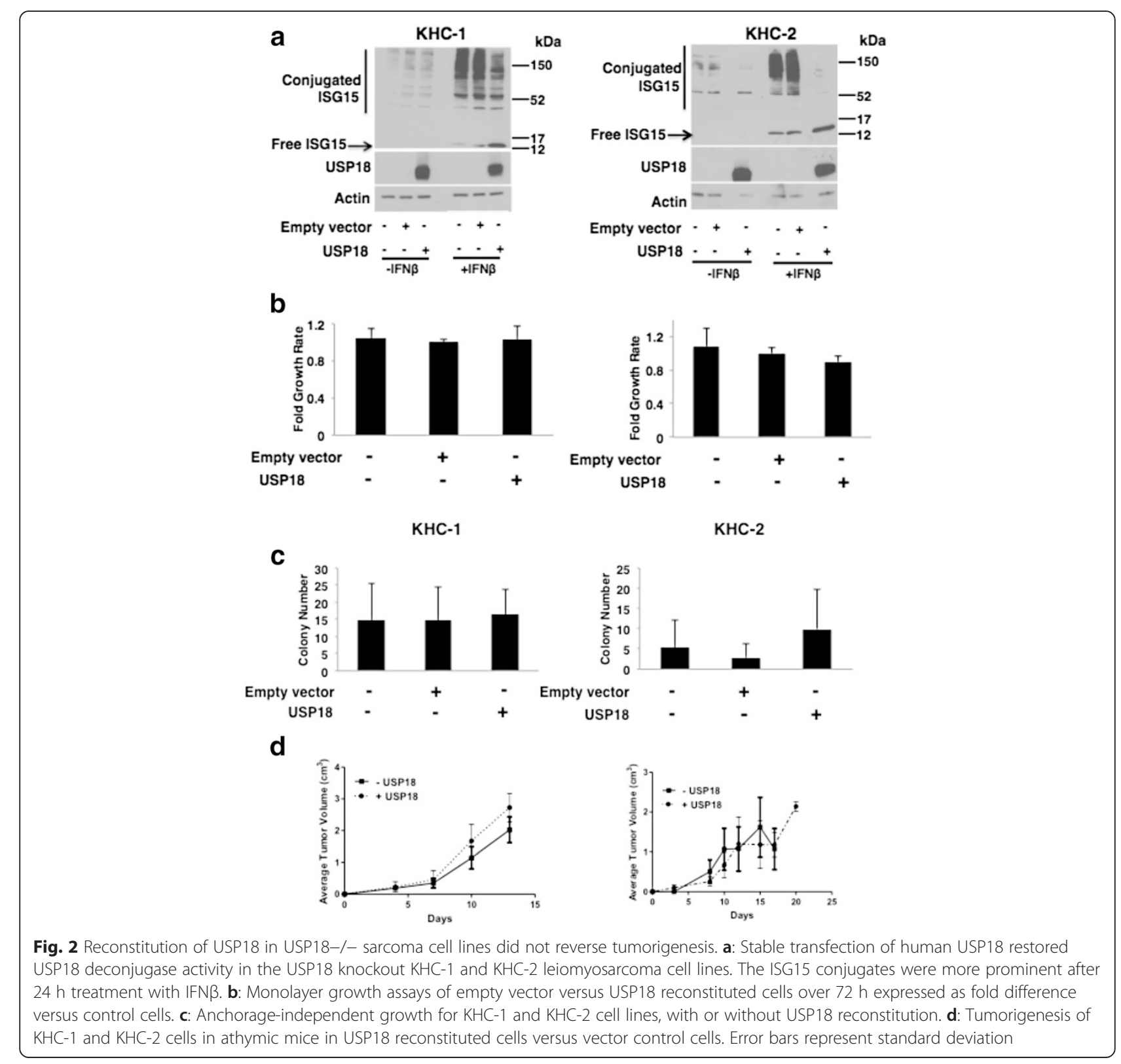


(Additional file 1: Table S2). Specimen site is indicated since samples were from a mix of primary tumors, metastases and tumors that had recurred. A representative immunohistochemical analysis of USP18 levels in histopathologically normal human lung and in clinical leiomyosarcomas is displayed in Fig. 5a and b. Findings revealed prominent USP18 immunostaining in lung macrophages (Fig. 5a) and also in histopathologically normal vascular smooth muscle cells with reduced expression relative to the adjacent leiomyosarcoma (Fig. 5b). Some leiomyosarcomas expressed
USP18 levels approaching that of histologically normal smooth muscle (Fig. 5b center panel). Vascular smooth muscle is the cell of origin for some leiomyosarcomas [37, 38].

To extend this analysis, USP18 levels were examined in a human leiomyosarcoma tissue microarray. Compared to normal tissues where USP18 staining was high, (vascular smooth muscle cells served as a control), there was a subgroup of leiomyosarcomas with reduced USP18 immunostaining (Fig. 5c). Clinical data were available for 89

a

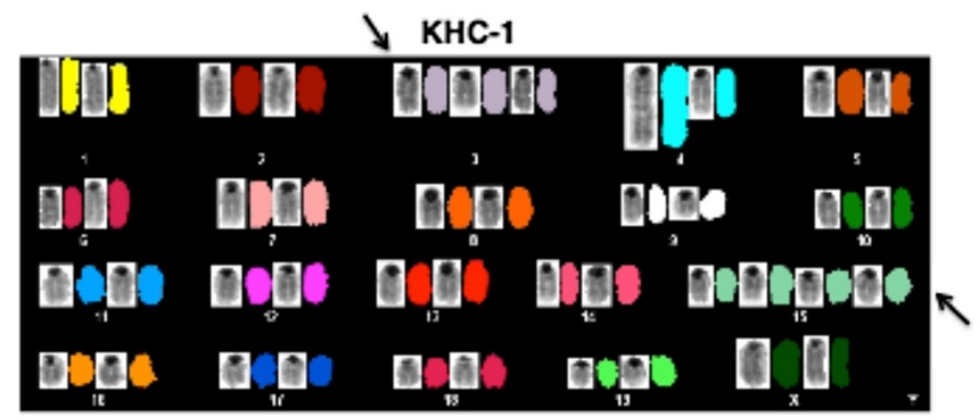

b

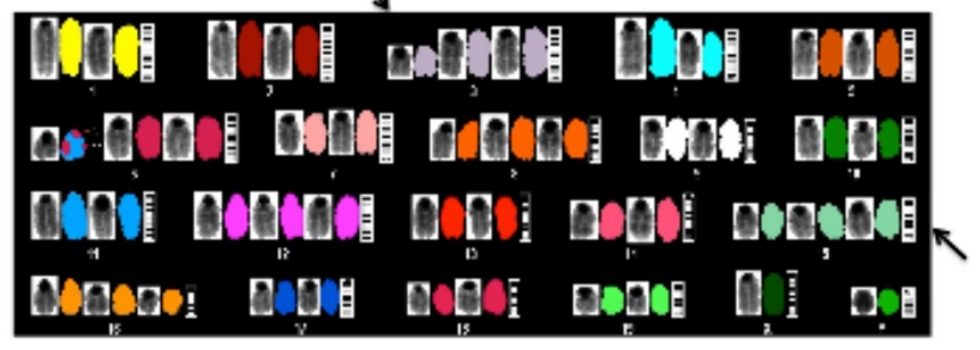

C

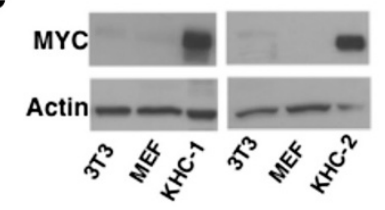

e KHC-1

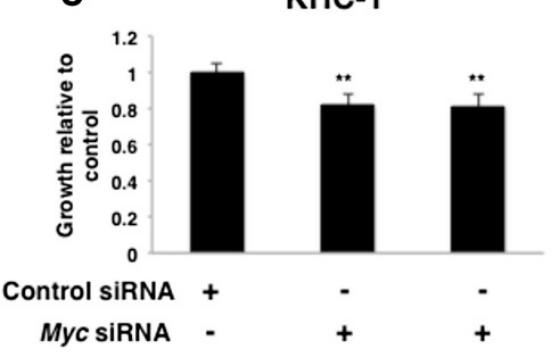

d

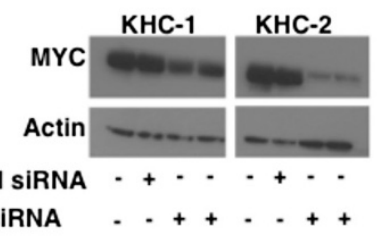

f

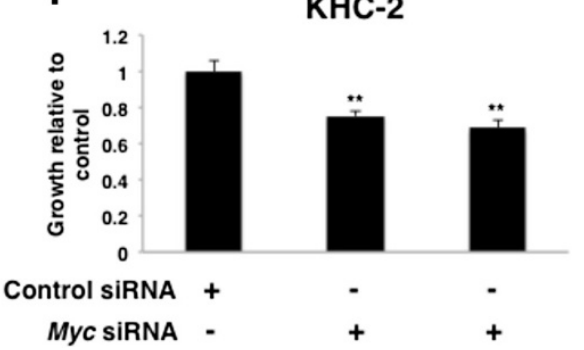

Fig. 3 Leiomyosarcoma cell lines are aneuploid and overexpress MYC. a and $\mathbf{b}$ : Cytogenetic analyses showed that both KHC-1 and KHC-2 had polysomy of chromosomes 3 and 15 (black arrows). c: Immunoblot analysis of MYC protein as shown for KHC-1 and KHC-2 cell lines as compared to NIH-3T3L1 and mouse embryonic fibroblast (MEF) cells. d: Immunoblot analysis of MYC levels validated knockdown by two independent siRNAs in KHC-1 and KHC-2 cells. e and $\mathbf{f}$ : Analysis of cell growth in KHC-1 and KHC-2 cells transfected with MYC or control siRNAs 


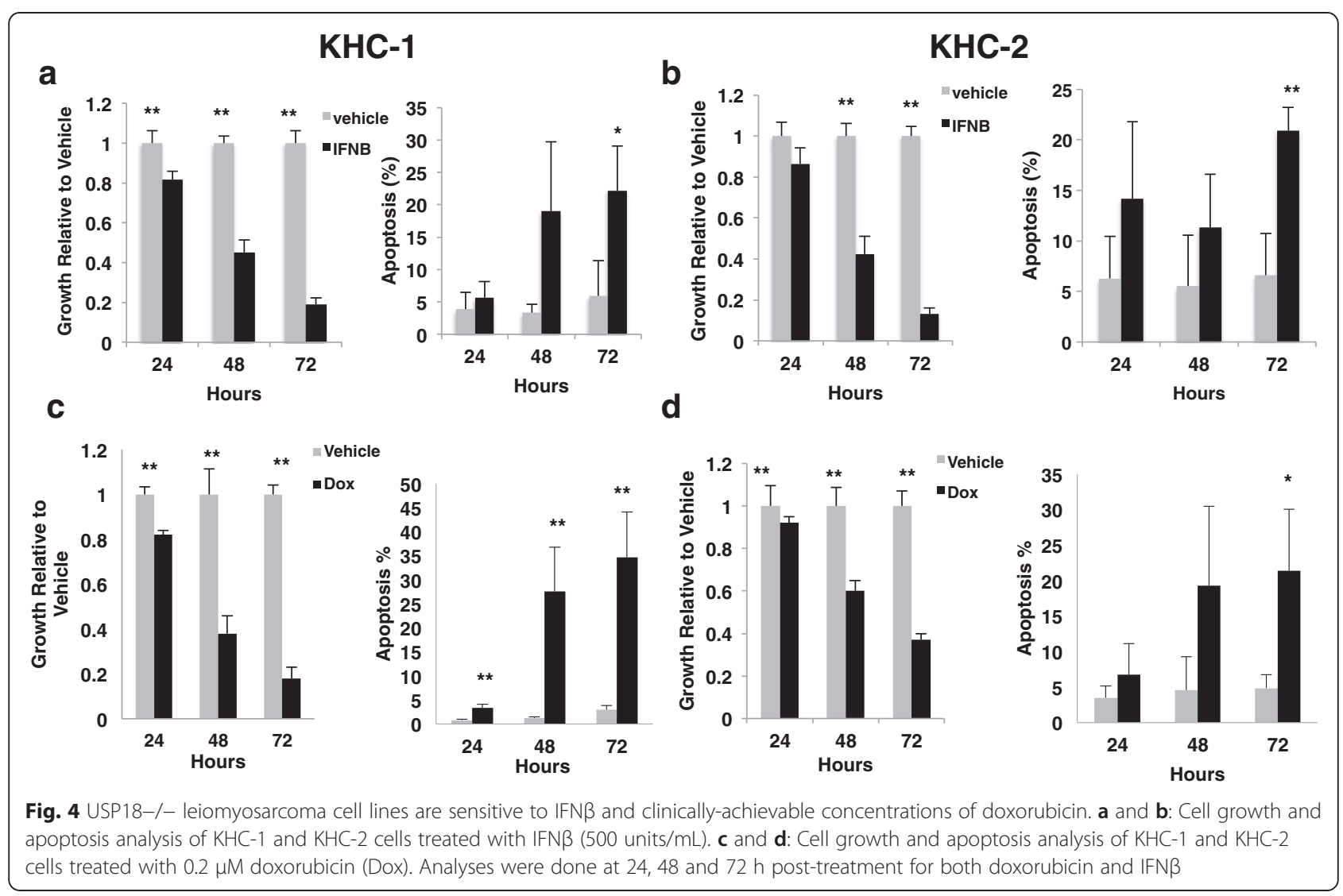

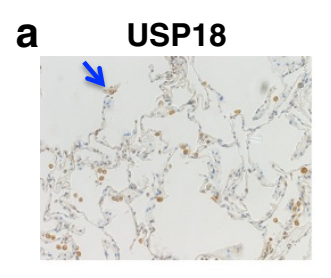

C

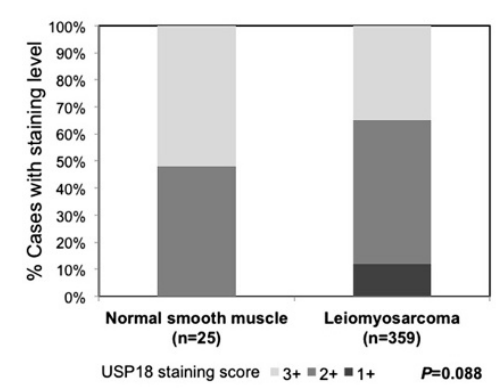

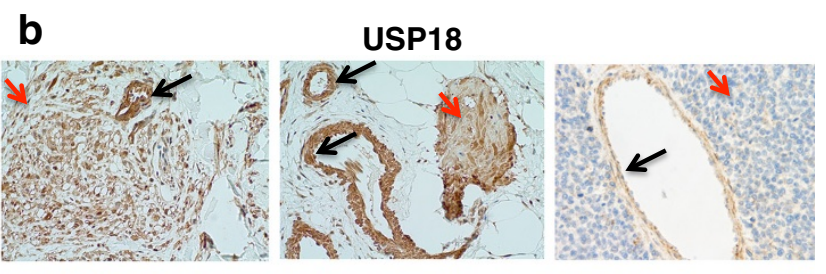

d

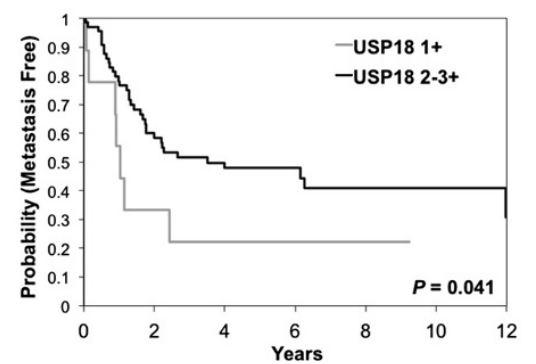

Fig. 5 Immunohistochemical analyses in human leiomyosarcoma cases and normal vascular smooth muscle and outcomes analysis. a: USP18 staining of normal lung tissue. Blue arrow indicates a USP18 positive macrophage. b: USP18 staining of clinical leiomyosarcoma with adjacent normal smooth muscle tissue. Abundant USP18 expression in vascular smooth muscle cells is highlighted with black arrows. Red arrows indicate leiomyosarcoma. c: Relative levels of USP18 in normal smooth muscle and in a series of leiomyosarcoma. $\mathbf{d}$ : Time to development of first metastasis for 89 primary uterine and soft tissue leiomyosarcoma patients 
primary uterine and soft tissue leiomyosarcomas. In univariable Cox proportional hazards analysis, retained USP18 expression was associated with prolonged time to first metastasis (HR 0.4543, 95 \% CI 0.2107-0.9795, $p=0.0441$ ) (Fig. $5 \mathrm{~d}$ ). USP18 levels were not predictive of overall survival (OS) $(p=0.72)$ or disease free survival (DSS) $(p=0.3371)$ (data not shown). Multivariate analysis was performed including factors shown in prior analyses as clinically prognostic in leiomyosarcoma [32]. In the current analysis, USP18 retained expression predicted longer time to metastasis $(p=0.0213)$.

\section{Discussion}

Leiomyosarcomas have complex karyotypes and treatment options are limited [39, 40]. Clinically-relevant murine leiomyosarcoma models should help develop innovative therapies for these sarcomas. Few soft-tissue murine models of leiomyosarcoma exist. Inactivation of PTEN in smooth muscle cells produced small mice with smooth muscle hyperplasia affecting blood vessels and the urinary and intestinal tracts, but not in the uterus [41]. Expression of the T antigens of the SV40 early region resulted in a narrow tissue-specific distribution of tumors. All female animals developed large uterine leiomyosarcomas by 3 months and most males exhibited enlarged seminal vesicles from smooth muscle hyperplasia [42]. In an experiment to specifically inactivate BRCA1, P53 and Rb in murine ovarian epithelial cells, tumors arose instead from adjacent smooth muscle and were diagnosed as leiomyosarcomas [43]. Thus, distinct genetic changes can cause leiomyosarcomas to arise from different primary sites.

The 3-MCA-carcinogen murine fibrosarcoma model provided insights into tumor immunology and cancer immunoediting. Mice with specific immune defects are particularly susceptible to chemical-induced carcinogenesis. Perforin-1, IFN- $\gamma$, STAT-1 and T cells each contribute to reducing 3-MCA-induced tumor formation and growth [44]. The immune system can drive tumors into a dormant state, which in turn affects immunoediting and tumorigenicity, as reviewed [44].

Defects in IFN signaling can augment tumorigenesis in the 3-MCA-sarcoma model; evidence from USP18 null mice identified an amplified IFN response as compared to wild-type mice, which is thought to provide a less permissive environment for tumors [45]. To directly explore the role of IFN signaling in leiomyosarcoma development it would prove informative in the future to cross the USP18 null mice with mice lacking either IFN or the IFN receptor (IFNGR1). However, transplantation of KHC-1 cells into immunocompentent mice did not result in a prominent immune cell response (data not shown), arguing against a driving role for the immune system in suppressing development of these leiomyosarcomas.
Abundant USP18 immunostaining in vascular smooth muscle cells implied that sarcomas seen in these mice could be of vascular origin. USP18 expression levels in clinical leiomyosarcoma varied widely with some staining at levels comparable to vascular smooth muscle and some with much lower levels (Fig. 5a and Additional file 1: Table S2). A significant association between USP18 levels and time to onset of metastasis was determined confirming a link between USP18 levels and disease progression, as shown in Fig. 5.

Sarcomas were not seen in the original report of USP18 null mice likely because this was in the C57/Bl6 and 129 mixed strain background. These mice developed neurological symptoms that reduced their survival to less than 20 weeks of age [21]. In marked contrast, USP18 null mice in this study had been bred onto the FVB/N strain background and rarely exhibited neurological symptoms.

USP18 null mice developed leiomyosarcomas with complex karyotypes and distinct genetic alterations reminiscent of human leiomyosarcomas. Common alterations included MYC overexpression and P53 stabilization. The genetic variability of leiomyosarcoma makes it essential to have different models in which to test antineoplastic drug treatments. Loss of PTEN sensitized leiomyosarcoma cells to rapamycin treatment [41], however tumors with different driver mutations will likely have different drug sensitivities.

Loss of USP18 activity is associated with type 1 IFN hypersensitivity [21]. Both murine USP18 null and human leiomyosarcoma cell lines described here responded independently to doxorubicin and type 1 IFN treatments. Activating the IFN pathway either directly with IFN or with an IFN-inducing agent might target leiomyosarcomas. Murine leiomyosarcoma cell lines also exhibited constitutive activation of phosphorylated STAT3 (pSTAT3). JAK2-STAT3 inhibitor treatment of these sarcoma cells led to downregulation of pSTAT3 and growth inhibition, indicating this type of inhibitor presents a potential treatment for leiomyosarcomas (Additional file 1: Figure. S2).

USP18 is the major deconjugase for ISG15, a type 1 IFNregulated ubiquitin-like protein modifier [18]. Decrease in USP18 has not been previously associated with sarcoma development. Conversely, USP18 repression has been associated with antineoplastic effects in lung and kidney cancers and in acute promyelocytic leukemia [26-28]. Immunohistochemical analysis of human tissues showed high levels of USP18 expression in normal vascular smooth muscle cells from which these tumors are likely derived [38]. IFN is known to stimulate vascular smooth muscle cell proliferation via PI3K and mTOR signaling [46]. The IFNhypersensitive environment found in USP18 null mice might deregulate proliferation of these vascular cells, initiating leiomyosarcoma formation. This possibility is consistent 
with the leiomyosarcoma formation seen when PI3K/ mTOR signaling is inappropriately activated in smooth muscle cells by deletion of the negative regulator, PTEN [41].

\section{Conclusions}

This study reports a new murine model of spontaneous leiomyosarcoma with associated transplantable cell lines. The tumors exhibited many features of human leiomyosarcomas including aneuploidy, MYC amplification and P53 stabilization. Sarcomas rapidly developed after transplantation of murine leiomyosarcoma cell lines into recipient mice, making this a tractable model for testing innovative leiomyosarcoma therapies. Taken together, these models can provide insights into both leiomyosarcoma biology and therapy.

\section{Additional files}

Additional file 1: Table S1. Antibodies used for immunohistochemistry studies. Table S2. Immunohistochemical staining of USP18 in clinical leiomyosarcoma samples. Figure S1. A: Dystrophic calcifications in USP18 null mice. A representative image of a USP18-/- mouse is shown. B: KHC-2 cells with reconstituted USP18 expression maintained expression for the duration of growth in mice. Immunoblot analysis of protein isolated from 4 control and 4 USP18 overexpressing independent orthotopic sarcomas harvested from mice. The immunoblot showed representative analysis of KHC-2 cells and this finding was also seen in KHC-1 cells (data not shown). Figure S2. USP18 null leiomyosarcoma cell lines are sensitive to treatment with the JAK2-STAT3 inhibitor, JSI-124. A: Immunoblot analysis of pSTAT3, STAT3, CDK4 and USP18 levels in $\mathrm{KHC}-1$ cells with and without stably restored USP18 activity. B: Growth analysis of KHC-1 cells with JAK2-STAT3 inhibitor, JSI-124. Similar effects were seen in KHC-2 cells (data not shown). Validation of JSI-124 repression of JAK2-STAT3 pathway C: Immunoblot analyses of phosphorylated JAK2 (pJAK2), JAK2, and actin with relative level of pJAK2/JAK2 calculated relative to control. D: Immunoblot analysis of phosphorylated STAT3 (pSTAT3), STAT3, cyclin D1 and actin levels. Figure S3. USP18 null leiomyosarcoma cell line KHC-1 and human leiomyosarcoma cell line SK-LMS-1 growth in response to interferon- $\beta$ (500Units/ml IFNB) or doxycycline (0.2 $\mu \mathrm{M}$ Dox) treatment over 3 days. Results expressed as fold relative to vehicle treated cells. Each experiment was performed in triplicate 3 separate times. Figure S4. USP18 null leiomyosarcoma cell line KHC-1 with restored USP18 expression did not affect response to IFNb (500Units/ml). Results expressed as fold relative to vehicle treated cells. (N.S. $=$ not significant). (PPTX $2131 \mathrm{~kb}$ )

Additional file 2: Mice Null for the Deubiquitinase USP18 Spontaneously Develop Leiomyosarcoma. (DOCX $115 \mathrm{~kb}$ )

\section{Competing interests}

The authors declare that they have no competing interests.

\section{Authors' contributions}

FC developed the animal model and cell lines, performed in vitro and in vivo experiments and wrote the manuscript; ED and SJF participated in the design of the study and edited the manuscript; YL, DJS and MK performed in vitro assays; AJG and PJH were involved with the immunohistochemical charaterization of the murine tumors; SSB carried out the karyotypic analysis of the murine cell lines; BLE, WAW, EGD, AJL, KET and VM located and analyzed the human leiomyosarcoma samples. TM and AS participated in subcutaneous tumor experiments. All authors read and approved the final manuscript.

\section{Acknowledgements}

The flow cytometry was carried out in Dartlab, the Immunoassay and the Flow Cytometry Shared Resource at the Geisel School of Medicine at Dartmouth. We thank Dr. Jacqueline Smith and Gary Ward for analysis of data and technical assistance. Research reported in this publication was supported by National Institutes of Health $(\mathrm{NIH})$ and National Cancer Institute (NCI) grants R01CA087546 (E. Dmitrovsky), R01-CA062275 (E. Dmitrovsky), and R01-CA190722 (E. Dmitrovsky), by a Samuel Waxman Cancer Research Foundation (SWCRF) grant (E. Dmitrovsky), by a UT-STARS award (E. Dmitrovsky) and by a grant from Uniting Against Lung Cancer with Mary Jo's Fund to Fight Cancer (S.J. Freemantle). E. Dmitrovsky is an American Cancer Society Professor supported by a generous gift from the F.M. Kirby Foundation. The G-banding and SKY analysis was provided by the Center for Cancer Research, $\mathrm{NCl}, \mathrm{NIH}$.

\section{Author details}

'Department of Pharmacology and Toxicology, Dartmouth, Hanover, $\mathrm{NH}$, USA. ${ }^{2}$ Department of Medicine, Dartmouth, Hanover, NH, USA. ${ }^{3}$ Department of Pathology, Dartmouth, Hanover, NH, USA. ${ }^{4}$ Department of Surgery, Dartmouth, Hanover, NH, USA. ${ }^{5}$ Norris Cotton Cancer Center, Lebanon, NH, USA. ${ }^{6}$ Geisel School of Medicine, Dartmouth, Hanover, NH, USA.

${ }^{7}$ Dartmouth-Hitchcock Medical Center, Lebanon, NH, USA. ${ }^{8}$ Thayer School of Engineering, Dartmouth College, Hanover, NH, USA. ${ }^{9}$ Comparative Molecular Cytogenetics Core, Mouse Cancer Genetics Program, National Cancer Institute, Frederick, MD, USA. ${ }^{10}$ Department of Pathology, The Mount Sinai Hospital, New York, NY, USA. "'Department of Pathology, The University of Texas MD Anderson Cancer Center, Houston, TX, USA. ${ }^{12}$ Sarcoma Research Center, The University of Texas MD Anderson Cancer Center, Houston, TX, USA. ${ }^{13}$ Department of Surgical Oncology, The University of Texas MD Anderson Cancer Center, Houston, TX, USA. ${ }^{14}$ Present address: MD Anderson Cancer Center, Houston, TX 77030-4009, USA.

Received: 19 February 2015 Accepted: 30 October 2015

Published online: 10 November 2015

\section{References}

1. Taylor BS, Barretina J, Maki RG, Antonescu CR, Singer S, Ladanyi M. Advances in sarcoma genomics and new therapeutic targets. Nat Rev Cancer. 2011;11(8):541-57.

2. Coindre JM, Terrier P, Guillou L, Le Doussal V, Collin F, Ranchere D, et al. Predictive value of grade for metastasis development in the main histologic types of adult soft tissue sarcomas: a study of 1240 patients from the French Federation of Cancer Centers Sarcoma Group. Cancer. 2001;91(10):1914-26.

3. Weitz J, Antonescu CR, Brennan MF. Localized extremity soft tissue sarcoma: improved knowledge with unchanged survival over time. J Clin Oncol. 2003;21(14):2719-25.

4. Wibmer C, Leithner A, Zielonke N, Sperl M, Windhager R. Increasing incidence rates of soft tissue sarcomas? A population-based epidemiologic study and literature review. Ann Oncol. 2010;21(5):1106-11.

5. ESMO / European Sarcoma Network Working Group. Soft tissue and visceral sarcomas: ESMO Clinical Practice Guidelines for diagnosis, treatment and follow-up. Ann Oncol. 2012;23 Suppl 7:vii92-9.

6. Ray-Coquard I, Thomas D. Targeted therapies: pazopanib for soft-tissue sarcoma: a PALETTE of data emerges. Nat Rev Clin Oncol. 2012;9(8):431-2.

7. Van Glabbeke M, van Oosterom AT, Oosterhuis JW, Mouridsen H, Crowther D, Somers R, et al. Prognostic factors for the outcome of chemotherapy in advanced soft tissue sarcoma: an analysis of 2,185 patients treated with anthracycline-containing first-line regimens-a European Organization for Research and Treatment of Cancer Soft Tissue and Bone Sarcoma Group Study. J Clin Oncol. 1999;17(1):150-7.

8. O'Sullivan B, Bell R, Bramwell V. Sarcoma of the soft tissues. In: Oxford Textbook of Oncology (ed 2). Edited by in Souhami RL, Tannock I, Hohenberger P. Oxford, United Kingdom: Oxford University Press, 2002:2495-2523.

9. Lorigan P, Verweij J, Papai Z, Rodenhuis S, Le Cesne A, Leahy MG, et al. European Organisation for Research and Treatment of Cancer Soft Tissue and Bone Sarcoma Group Study: Phase III trial of two investigational schedules of ifosfamide compared with standard-dose doxorubicin in advanced or metastatic soft tissue sarcoma: a European Organisation for Research and Treatment of Cancer Soft Tissue and Bone Sarcoma Group Study. J Clin Oncol. 2007;25(21):3144-50. 
10. Helman $L$, Meltzer P. Mechanisms of sarcoma development. Nat Rev Cancer. 2003;3(9):685-94.

11. Borden EC, Baker LH, Bell RS, Bramwell V, Demetri GD, Eisenberg BL, et al. Soft tissue sarcomas of adults: state of the translational science. Clin Cancer Res. 2003;9(6):1941-56.

12. Perot G, Chibon F, Montero A, Lagarde P, de The H, Terrier P, et al. Constant p53 pathway inactivation in a large series of soft tissue sarcomas with complex genetics. Am J Pathol. 2010;177(4):2080-90.

13. Ito M, Barys L, O'Reilly T, Young S, Gorbatcheva B, Monahan J, et al. Comprehensive mapping of p53 pathway alterations reveals an apparent role for both SNP309 and MDM2 amplification in sarcomagenesis. Clin Cancer Res. 2011;17(3):416-26.

14. Dodd RD, Mito JK, Kirsch DG. Animal models of soft-tissue sarcoma. Dis Model Mech. 2010:3(9-10):557-66.

15. Afify A, Mark HF. Trisomy 8 in embryonal rhabdomyosarcoma detected by fluorescence in situ hybridization. Cancer Genet Cytogenet. 1999;108(2):127-32.

16. Selvarajah S, Yoshimoto M, Prasad M, Shago M, Squire JA, Zielenska M, et al. Characterization of trisomy 8 in pediatric undifferentiated sarcomas using advanced molecular cytogenetic techniques. Cancer Genet Cytogenet. 2007;174(1):35-41.

17. Tsiatis AC, Herceg ME, Keedy VL, Halpern JL, Holt GE, Schwartz HS, et al. Prognostic significance of C-Myc expression in soft tissue leiomyosarcoma. Mod Pathol. 2009:22(11):1432-8

18. Malakhov MP, Malakhova OA, Kim KI, Ritchie KJ, Zhang DE. UBP43 (USP18) specifically removes ISG15 from conjugated proteins. J Biol Chem. 2002;277(12):9976-81.

19. Farrell PJ, Broeze RJ, Lengyel P. Accumulation of an mRNA and protein in interferon-treated Ehrlich ascites tumour cells. Nature. 1979;279(5713):523-5.

20. Loeb KR, Haas AL. The interferon-inducible 15-kDa ubiquitin homolog conjugates to intracellular proteins. J Biol Chem. 1992;267(11):7806-13.

21. Ritchie KJ, Malakhov MP, Hetherington CJ, Zhou L, Little MT, Malakhova OA, et al. Dysregulation of protein modification by ISG15 results in brain cell injury. Genes Dev. 2002;16(17):2207-12.

22. Ketscher L, Hannss R, Morales DJ, Basters A, Guerra S, Goldmann T, et al. Selective inactivation of USP18 isopeptidase activity in vivo enhances ISG15 conjugation and viral resistance. Proc Natl Acad Sci U S A. 2015;112(5):1577-82.

23. Malakhova OA, Yan M, Malakhov MP, Yuan Y, Ritchie K, Kim Kl, et al. Protein ISGylation modulates the JAK-STAT signaling pathway. Genes Dev. 2003;17(4):455-60.

24. Zhang X, Bogunovic D, Payelle-Brogard B, Francois-Newton V, Speer SD, Yuan C, et al. Human intracellular ISG15 prevents interferon-alpha/beta over-amplification and auto-inflammation. Nature. 2015;517(7532):89-93.

25. Duex JE, Sorkin A. RNA interference screen identifies Usp18 as a regulator of epidermal growth factor receptor synthesis. Mol Biol Cell. 2009;20(6):1833-44.

26. Guo Y, Dolinko AV, Chinyengetere F, Stanton B, Bomberger JM, Demidenko E, et al. Blockade of the ubiquitin protease UBP43 destabilizes transcription factor PML/RARalpha and inhibits the growth of acute promyelocytic leukemia. Cancer Res. 2010;70(23):9875-85.

27. Guo Y, Chinyengetere F, Dolinko AV, Lopez-Aguiar A, Lu Y, Galimberti F, et al. Evidence for the ubiquitin protease UBP43 as an antineoplastic target. Mol Cancer Ther. 2012;11(9):1968-77.

28. Shahidul Makki M, Cristy Ruteshouser E, Huff V. Ubiquitin specific protease 18 (Usp18) is a WT1 transcriptional target. Exp Cell Res. 2013;319(5):612-22.

29. Richer E, Prendergast C, Zhang DE, Qureshi ST, Vidal SM, Malo D. N-ethyl-Nnitrosourea-induced mutation in ubiquitin-specific peptidase 18 causes hyperactivation of IFN-alphaaß signaling and suppresses STAT4-induced IFN-gamma production, resulting in increased susceptibility to Salmonella typhimurium. J Immunol. 2010;185(6):3593-601.

30. Liu X, Sempere LF, Galimberti F, Freemantle SJ, Black C, Dragnev KH, et al. Uncovering growth-suppressive microRNAs in lung cancer. Clin Cancer Res. 2009;15(4):1177-83

31. Langenfeld J, Lonardo F, Kiyokawa H, Passalaris T, Ahn MJ, Rusch V, et al. Inhibited transformation of immortalized human bronchial epithelial cells by retinoic acid is linked to cyclin E down-regulation. Oncogene. 1996;13(9):1983-90.

32. Demicco EG, Boland GM, Brewer Savannah K, Lusby K, Young ED, Ingram $D$, et al. Progressive loss of myogenic differentiation in leiomyosarcoma has prognostic value. Histopathology. 2014;66:627-38.

33. Kilpatrick SE, Mentzel T, Fletcher CD. Leiomyoma of deep soft tissue. Clinicopathologic analysis of a series. Am J Surg Pathol. 1994;18(6):576-82.
34. Donehower LA, Harvey M, Slagle BL, McArthur MJ, Montgomery Jr CA, Butel JS, et al. Mice deficient for p53 are developmentally normal but susceptible to spontaneous tumours. Nature. 1992;356(6366):215-21.

35. Adamson MC, Dennis C, Delaney S, Christiansen J, Monkley S, Kozak CA, et al. Isolation and genetic mapping of two novel members of the murine Wnt gene family, Wnt11 and Wnt12, and the mapping of Wnt5a and Wnt7a. Genomics. 1994;24(1):9-13.

36. Bowman T, Garcia R, Turkson J, Jove R. STATs in oncogenesis. Oncogene. 2000;19(21):2474-88.

37. Morel M, Taieb S, Penel N, Mortier L, Vanseymortier L, Robin YM, et al. Imaging of the most frequent superficial soft-tissue sarcomas. Skeletal Radiol. 2011;40(3):271-84.

38. Salemis NS. Recurrent subcutaneous trunk leiomyosarcoma: Management and review of the literature. J Nat Sci Biol Med. 2013;4(1):238-42.

39. Edris B, Weiskopf K, Volkmer AK, Volkmer JP, Willingham SB, ContrerasTrujillo $\mathrm{H}$, et al. Antibody therapy targeting the CD47 protein is effective in a model of aggressive metastatic leiomyosarcoma. Proc Natl Acad Sci U S A. 2012;109(17):6656-61.

40. Evans H, Shipley J: Pathology and Genetics of Tumours of Soft Tissue and Bone-World Health Organization Classification of Tumours. In Edited by Fletcher CDM, Unni KK, Mertens F (IARC Press, Lyon). France: IARC Press Lyon; 2002:131-134.

41. Hernando E, Charytonowicz E, Dudas ME, Menendez S, Matushansky I, Mills J, et al. The AKT-mTOR pathway plays a critical role in the development of leiomyosarcomas. Nat Med. 2007;13(6):748-53.

42. Politi K, Szabolcs M, Fisher P, Kljuic A, Ludwig T, Efstratiadis A. A mouse model of uterine leiomyosarcoma. Am J Pathol. 2004;164(1):325-36.

43. Clark-Knowles KV, Senterman MK, Collins O, Vanderhyden BC. Conditional inactivation of Brca1, p53 and Rb in mouse ovaries results in the development of leiomyosarcomas. PLoS One. 2009;4(12):e8534.

44. Schreiber TH, Podack ER. A critical analysis of the tumour immunosurveillance controversy for 3-MCA-induced sarcomas. Br J Cancer. 2009:101(3):381-6.

45. Burkart C, Arimoto K, Tang T, Cong X, Xiao N, Liu YC, et al. Usp18 deficient mammary epithelial cells create an antitumour environment driven by hypersensitivity to IFN-lambda and elevated secretion of Cxcl10. EMBO Mol Med. 2013;5(7):967-82.

46. Mourani PM, Garl PJ, Wenzlau JM, Carpenter TC, Stenmark KR, Weiser-Evans MC. Unique, highly proliferative growth phenotype expressed by embryonic and neointimal smooth muscle cells is driven by constitutive Akt, mTOR, and p70S6K signaling and is actively repressed by PTEN. Circulation. 2004;109(10):1299-306.

\section{Submit your next manuscript to BioMed Central and take full advantage of:}

- Convenient online submission

- Thorough peer review

- No space constraints or color figure charges

- Immediate publication on acceptance

- Inclusion in PubMed, CAS, Scopus and Google Scholar

- Research which is freely available for redistribution

Submit your manuscript at www.biomedcentral.com/submit
C Biomed Central 